\title{
Antimicrobial Property of Hydrocolloid Impression Material Incorporated with Silver Nanoparticles Against Staphylococcus Aureus
}

\author{
Norbu Penden Wangchuk ${ }^{1}$, Passiri Nisalak ${ }^{1}$,Boonyanit Thaweboon ${ }^{2}$, Sroisiri Thawboon ${ }^{2}$ and Pornrachanee Sawaengkit ${ }^{1}$ \\ ${ }^{1}$ Orthodontic Department, Faculty of Dentistry, Mahidol University, Bangkok 10400, Thailand \\ ${ }^{2}$ Oral Microbiology Department, Faculty of Dentistry, Mahidol University, Bangkok 10400, Thailand
}

\begin{abstract}
Dental impressions can easily become contaminated with patient's blood and saliva which are capable of transmitting infectious diseases to dental personnel. The addition of antimicrobial agents into impression materials could be effective in reducing the chances of cross-infection. Silver nanoparticles have been applied in dentistry as a potent antimicrobial agent. This study aims to evaluate the in vitro antimicrobial efficacy of silver nanoparticles incorporated to irreversible hydrocolloid impression material against Staphylococcus aureus. Silver nanoparticles $\left(\mathrm{AgZrPO}_{4}\right.$, National Direct Network Company, Thailand) at concentrations of $0.25 \%, 0.50 \%, 1.00 \%$ and $1.50 \% \mathrm{w} / \mathrm{w}$ were added to powder of impression materials (Kromopan, Lascod, Ilaty). Impression material samples were prepared on sterile plate in accordance with manufacturer's instruction. After setting, a 100 microliter of S. aureus ATCC6538 suspension $\left(10^{6} \mathrm{cells} / \mathrm{mL}\right)$ were inoculated on the surface of the impression sample and left for 10 minutes. The amount of $S$. aureus on the surface was quantified using imprint technique on Mannitol Salt agar. Impression materials incorporated with $\mathrm{AgZrPO}_{4}$ showed antimicrobial property against $S$. aureus (up to $95 \%$ reduction) compared with control (impression material without $\mathrm{AgZrPO}_{4}$ ). Even though the mechanism of antimicrobial action was not clearly understood, $\mathrm{AgZrPO}_{4}$ incorporated to impression material was demonstrated to possess an inhibitory effect against pathogenic bacteria. Further studies are needed to investigate physical properties of the material and the clinical usage.
\end{abstract}

\section{Introduction}

The study of dental biomaterials and its properties are of immense interest in the field of research and innovative technology. One of the most widely accepted and used in dentistry is the hydrocolloid alginate impression material owing to its several advantages over the others [1]. Impression material has been one of the many biomaterials that have captured the attention of many researchers. Firstly in trying to replicate the anatomic details of the oral cavity and then further to addition of antimicrobial agent to render it sterile. The reasons for the purpose of rendering it sterile erupts from the fact that dental impressions become contaminated with patient's saliva and/or blood that can consequently crosscontaminate stone casts poured against them. Subsequent handling puts the dental personnel at high risks of microbial transmission/infection.

The micro flora of the oral cavity has a diverse community of microbes ranging from bacteria to fungi with studies even confirming the presence of viruses [2]. In a three year retrospective study by McCormack et al, they reported a large data regarding the recovery of Staphylococcus aureus in the oral and perioral cavity [3].

His findings condemned oral rinse as the main specimen from which the $S$. aureus could be isolated. It warns us of the potential cross-infection and dissemination to other body sites. It may be indispensable to the hands of dentists but impression taking is also known for effective cross infection and transmission of microbes and diseases thereof like many other dental practices [4]. As it is known that the oral cavity is a host to many known and unknown microbes [2], researches have confirmed that the oral fluid diffuse into the impression material [5] suggesting a pathway for initiating a cross infection that can be potentially deadly in transmitting infectious microorganisms. Of course it is needless to mention the numerous methods invented to render the impression material sterile in the form of aerosol spraying or immersion in the disinfectant solution or solely rinsing under running tap water[6], [7]. These methods tend to have some shortcomings either in the form of disinfection achieved or the compromise resulting in the physical property of the impression material that is controversial overall [8].

The dawn of nanotechnology, silver nanoparticles to be specific has gained much popularity owing to its potent antimicrobial activity [9]. Its use is well documented especially as a dressing material resulting from injury at times of battle. Dentistry also reports its use in various field including prosthetic dentistry, dental 
implants and restorative as well as in endodontics in the form of root canal irrigation solution [10].

The purpose of this study is therefore to evaluate the antimicrobial property of hydrocolloid impression material incorporated with silver zirconium phosphate nanoparticles against $S$. aureus.

\section{Materials and methods}

\subsection{Microorganisms}

Staphylococcus aureus ATCC 5638 were obtained from the culture collection of the Department of Oral Microbiology, Faculty of Dentistry, Mahidol University, Thailand. They were grown in Mannitol Salt agar at 30 to $35{ }^{\circ} \mathrm{C}$ for 7 days. Microbial suspension was prepared in saline solution to approximately $10^{8} \mathrm{CFU} / \mathrm{ml}$ using Mac Farlands no. 0.5 and further diluted to $10^{5} \mathrm{CFU} / \mathrm{ml}$ before being used in the antimicrobial testing.

\subsection{Irreversible hydrocolloid impression material}

The irreversible alginate impression material used for the test was Kromopan class A, type I dust free complying with ISO 1563. Lascod, Italy.

\subsection{Silver based nanoparticles}

The silver zirconium phosphate nanoparticles $\left(\mathrm{AgZrPO}_{4}\right)$ were supplied from the National Direct Network Company, Thailand and were mixed with powder of hydrocolloid impression material to the concentrations of $0.25 \%, 0.50 \%, 1.00 \%$ and $1.50 \% \mathrm{w} / \mathrm{w}$.

\subsection{Antimicrobial testing}

The irreversible hydrocolloid impression material powder incorporated with each concentration of silver nanoparticles $(0.25 \%, 0.50 \%, 1.00 \%$, and $1.50 \% \mathrm{w} / \mathrm{w})$ was mixed with water following the manufacturer's instruction and then poured into sterile petri dish. The 100 microliter of the microbial suspension were then spread on the surface of the impression material and left for 10 minutes. A $2 \times 2 \mathrm{~cm}^{2}$ sterile Whatmans filter paper was then placed on the spread bacteria and left for few minutes. This step simulates the activity of microbes that are harvested during the impression taking.

The filter paper were then removed and placed in the Mannitol Salt agar plate which serves as a selective media for the growth of $S$. aureus and was incubated at $37{ }^{\circ} \mathrm{C}$ for 48 hours. This procedure best describes the optimal technique that could give quantitative results by the growth of the $S$. aureus, commonly known as imprint technique. Hydrocolloid impression material without $\mathrm{AgZrPO}_{4}$ served as control. The number of bacterial growth recovered from the media will represent the antibacterial activity.

All the experiments were done in triplicate in three different occasions.

\subsection{Statistical analysis}

The antimicrobial property was evaluated by the recovery of number of $S$. aureus $\left(\mathrm{CFU} / \mathrm{cm}^{2}\right)$ on Mannitol Salt agar by imprint technique with non-parametric test using Kruskal-Wallis test. Pairwise comparison of group was done using Mann-Whitney test at a confidence interval set at $95 \%$ with the significance level set at $\alpha=0.05$.

Table 1. The mean number and standard deviation of $S$. aureus detected on impression material $\left(\mathrm{CFU} / \mathrm{cm}^{2}\right)$ by imprint method.

\begin{tabular}{|c|c|c|c|c|}
\hline \multicolumn{4}{|c|}{$\mathrm{AgZrPO}_{4}(\% \mathrm{w} / \mathrm{w})$} & \multirow[t]{2}{*}{ no $\mathrm{AgZrPO}_{4}$} \\
\hline 0.25 & 0.50 & 1.00 & 1.50 & \\
\hline $1.67 \pm 0.4$ & $0.65 \pm 0.53$ & $0.31 \pm 0.38$ & $0.13 \pm 0.28$ & $2.47 \pm 0.45$ \\
\hline
\end{tabular}

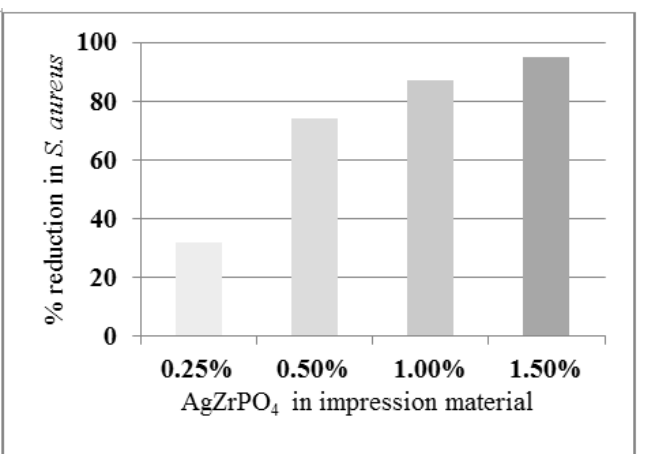

Figure 1. The percentage of $S$. aureus reduction at various concentration of $\mathrm{AgZrPO}_{4}$ containing impression material compared with control.

\section{Results}

Imprint technique was used for this method, as it is noninvasive easy-to-use that would give representative and quantitative results for samples of specific unit areas. In this study the results were collected as the observed number of bacterial growth in all the media reflecting the different concentrations of $\mathrm{AgZrPO}_{4}$ containing impression material. Table 1 shows the mean and standard deviation of viable bacteria recovered from impression material. It was evident to see that that least number of colonies $(0.31 \pm 0.38)$ was observed in the impression material with the highest concentration of $\mathrm{AgZrPO}_{4}(1.50 \%)$. On the other hand, the highest number of bacteria $(1.67 \pm 0.48)$ was recovered from the impression material containing the least $\mathrm{AgZrPO}_{4}$ $(0.25 \%)$ that was comparable to that of the bacteria recovered from the control group without the $\mathrm{AgZrPO}_{4}$. As seen in the Fig. 1, the percentage reduction of the viable bacteria is seen to increase in a dose dependent manner. The impression material containing $(0.25 \%)$ $\mathrm{AgZrPO}_{4}$ showed the least percentage reduction of $32 \%$. On the other hand, almost up to $95 \%$ reduction in the viable $S$. aureus was seen with impression material containing $(1.50 \%) \quad \mathrm{AgZrPO}_{4}$ that corresponds to its highest concentration used. Statistical analysis showed no significant difference when the control group was compared to that of the lowest concentration of the $\mathrm{AgZrPO}_{4}(0.25 \%)$. However, significant difference was 
seen among the different concentrations of $\mathrm{AgZrPO}_{4}$ containing impression material and that between the control and $0.50 \%, 1.00 \%$ and $1.50 \%$.

\section{Discussion}

Infection control and prevention is of paramount importance to prevent cross contamination. It has been a huge concern in the medical practice including dentistry. One of the pathways to cross-infection can be demonstrated simply by the use of a common biomaterial by the name, irreversible hydrocolloid impression material while making a negative replica of the patients intra-oral hard and soft tissue. This material owing to their composition and the nature of hydrophilic mechanism of solidification can be easily infected with microorganisms present in the oral cavity and therefore be responsible for cross-infection. Literature has it that the oral fluids diffuse into the impression material up to 540 micrometer establishing the fact that impression material could be contaminated with microorganisms from oral fluid [5], [11]. It is also reported that impression material when not disinfected can be heavily contaminated with microorganisms.

The earliest methods to disinfect the impression material included the rinsing of the impression under running tap water. Many methods have been developed and applied since then in an attempt to disinfect the impression material in the form of either mixing the disinfectant directly with the impression material or immersed into or sprayed in the form of aerosol. Potential disinfectants include iodophors, chlorhexidine, phenolics and inorganic ions such as copper or fluoride. Quaternary ammonium compounds are reported to have limited antimicrobial abilities. Although the above methods and the disinfectants were effective in reducing the number of bacteria, difficulties were faced when complying with the procedure of doing "another step" [12]. Moreover, immersion techniques are known to distort the anatomic details in addition to taking up much of the time before the impression material can be poured. Some of the agents could cause adverse tissue response such as the quaternary ammonium compounds when present in multiple forms. Aerosol spraying has limitations in reaching every nook and corner of the dental impression.

On the other hand, Silver nanoparticles were extensively explored for their antimicrobial activity. The earliest reports of silver as documented were used for wound dressings, catheters and prosthesis[13]. It has been extensively researched and experimented in various dental fields including endodontics, prosthetic dentistry as well as dental implants. And since then, its use evolved giving way to the latest technology of nanoparticles that had the advantage of small-sized particles showing efficient antimicrobial properties due to their larger surface area producing an effective contact with microorganism [14]. Our study demonstrated that $\mathrm{AgZrPO}_{4}$ when used in mixture with the impression material could have antimicrobial effects at concentrations as low as $0.25 \%$ against $S$. aureus. This result is in agreement with finding of Saengmee-
Anupharb et al where silver inorganic materials were evaluated for the antimicrobial activities including silver zeolite $(\mathrm{AgZ})$, silver zirconium phosphate $\left(\mathrm{AgZrPO} \mathrm{PO}_{4}\right)$ and silver zirconium phosphate silicate (AgZrPSi) [14]. However, this antimicrobial activity exhibited by $\mathrm{AgZrPO}_{4}$ is still an ambiguity. Reliable researches base the whole mechanism of antimicrobial activity firstly to the silver ions interaction with the cell wall, cell membrane and cell envelope of microorganism causing them to rupture and consequently resulting in cell death. It is also shown that silver ions do react with the nucleophilic amino acid residues in proteins that are attached to sulfhydryl, amino, imidazole, phosphate and carbonyl groups of membrane or enzyme proteins [13]. Some studies relate the antimicrobial property to the structural changes induced in the bacterial cell by formation of free radicals and subsequent free-radical induced membrane damage [15].

The results of this study, through the statistical analysis, found no significant difference in bacterial inhibition between the control and the $0.25 \% \mathrm{AgZrPO}_{4}$ containing impression material. Significant difference was found between the control and the $0.50 \%, 1.00 \%$ and with $1.50 \% \mathrm{AgZrPO}_{4}$ and also between the different concentration of $\mathrm{AgZrPO}_{4}$ containing impression material. With the increase in concentration of the nanoparticles in the impression material, their antimicrobial activity increased vice-versa. From this inference, although a high level of antibacterial activity can be achieved with the use of $1.50 \%$ of $\mathrm{AgZrPO}_{4}$ in the impression material, the percentage reduction of $S$. aureus is comparable to that $0.50 \% \quad \mathrm{AgZrPO}_{4}$ and therefore recommend the use of $0.50 \%$ of silver nanoparticle in impression material that will aim to achieve the maximum antimicrobial benefit while maintaining a low dose level of the disinfectant at the same time.

Few studies have reported the effect disinfection procedures had on the dimensional accuracy and surface quality of the irreversible hydrocolloid impression material. One study reports an increased distortion of irregular areas of the impression when using the immersion technique [8]. While there is a lack of evidence on the effect $\mathrm{AgZrPO}_{4}$ has on the physical property of the impression material, it was interesting to note that Bajrachary et al, in their study of Poly methyl methacrylate resin incorporated with silver nanoparticles did not compromise the flexural strength of the Poly methyl methacrylate resin [16]. The findings of Slane et al, in acrylic bone cement impregnated with silver nanoparticles authenticate the least effect it has on the materials physical property where it was established that under low concentrations of metallic silver nanoparticles, acrylic bone cements had retained their mechanical and material properties similar to the standard cement [17]. It may not be appropriate to say the physical properties of the impression material incorporated with $\mathrm{AgZrPO}_{4}$ will be least affected but rather be experimented and prove accordingly.

As with the problem of every antibacterial agent, silver nanoparticle has also expressed concern regarding 
the side effect especially in terms of cytotoxicity and genotoxicity. Although limited studies can be found that are aimed at investigating the genotoxicity of the nanoparticles, it is nevertheless considered complex. One study in particular points out that the most resistant organism to genotoxicity being the human cell cultures [18]. More over the overall time period of contact of the impression material with the oral mucosa and related structures is limited to few minutes. This is even supported by the fact that oral healthcare products do contain silver which is attributed to the broad-spectrum antimicrobial effects and low toxicity to humans [14]. This propels us to further research more regarding the potential ability to produce an impression material equipped with an antibacterial agent least harmful to the patient while exerting the most beneficial antibacterial activity.

Indeed the human oral cavity is extensively populated with a vast variety of microbes out of which $S$. aureus having reported a carriage rate of $24-84 \%$ in healthy oral cavity and $48 \%$ incidence among the denture-wearing population [19], takes the credit for causing majority of the deleterious clinical diseases involving the hard and soft tissue of the mouth [20]. Since its spread by contact is vividly documented in literature posing a great deal of threat to the dental personnel, this study therefore provides a significantly useful alternative to control the pathologies connected with $S$. aureus and other associated microbes. Dental impressions can be easily contaminated with patient's blood and saliva capable of transmitting infectious diseases to dental personnel. The method adopted in this experimental study was an attempt to produce an impression material with the addition of an antimicrobial agent so as to render the impression material effective against the numerous microbes including the $S$. aureus. The results obtained reflect the antimicrobial property of the $\mathrm{AgZrPO}_{4}$ in the impression material that was seen to be effective in a dose dependent manner.

In conclusion, hydrocolloid impression material containing $\mathrm{AgZrPO}_{4}$ could evidently reduce the number of test bacteria. However, further studies are required to test the accuracy in capturing the anatomic details including the physical properties with this impression material. Nevertheless, this basic research provides useful information on the application of $\mathrm{AgZrPO}_{4}$ in dental product to control or reduce risk of cross-infection. Although the invention of 3-D scanner and printer endanger the practice of using conventional alginate impression material, it is still nevertheless an economic, safe and reliable method of impression taking of the oral cavity in many of the developing countries

\section{Acknowledgement}

The authors would like to acknowledge the kind assistance generously offered by Miss Thaniya Maudcheingka including the other staff members of Oral
Microbiology Department, Faculty of Dentistry, Mahidol University for trying to make this research a success. We also extend our heartfelt gratitude towards the Faculty of Dentistry, Mahidol University, Thailand and the Ministry of Health, Royal Government of Bhutan for their financial and academic support.

\section{References}

1. T.E. Donovan, W.W. Chee, Dent Clin North Am 48, vi-vii, 445-70. (2004).

2. M. Avila, D.M. Ojcius, Ö. Yilmaz, DNA and Cell Biology 28, 405-411. (2009).

3. M.G. McCormack, A.J. Smith, A.N. Akram, M. Jackson, D. Robertson, G. Edwards, American Journal of Infection Control 43, 35-37. (2015).

4. H. Egusa, T. Watamoto, K. Abe, M. Kobayashi, Y. Kaneda, S. Ashida, T. Matsumoto, H. Yatani, Int J Prosthodont 21, 62-8. (2008).

5. R. Surna, J. Junevicius, E. Rutkauskas, Stomatologija 11, 129-34. (2009).

6. F.A. Rueggeberg, F.E. Beall, M.T. Kelly, G.S. Schuster, J Prosthet Dent 67, 628-31. (1992).

7. I.H. Rweyendela, M. Patel, C.P. Owen, Sadj 64, 208, 210-2. (2009).

8. A. Rentzia, D.C. Coleman, M.J. O'Donnell, A.H. Dowling, M. O'Sullivan, J Dent 39, 133-40. (2011).

9. K. Chaloupka, Y. Malam, A.M. Seifalian, Trends Biotechnol 28, 580-8. (2010).

10. J.M. Correa, M. Mori, H.L. Sanches, A.D. da Cruz, E. Poiate, Jr., I.A. Poiate, Int J Biomater 2015, 485275. (2015).

11. C.P. Owen, R. Goolam, Int J Prosthodont 6, 480-94. (1993).

12. D.A. Flanagan, C.J. Palenik, J.C. Setcos, C.H. Miller, Dental Materials 14, 399-404. (1998).

13. J.S. Kim, et al., Nanomedicine: Nanotechnology, Biology and Medicine 3, 95-101. (2007).

14. S. Saengmee-Anupharb, T. Srikhirin, B. Thaweboon, S. Thaweboon, T. Amornsakchai, S. Dechkunakorn, T. Suddhasthira, Asian Pac J Trop Biomed 3, 47-52. (2013).

15. M. Chen, Z. Yang, H. Wu, X. Pan, X. Xie, C. Wu, International Journal of Nanomedicine 6, 2873-2877. (2011).

16. S. Bajrachary, S. Thaweboon, B. Thaweboon, A. Wonglamsam, T. Sirthavaj, In vitro evaluation of candida albicans biofilm formation on denture base PMMA resin incorporated with silver nanoparticles and its effect on flexural strength, 2014, 51-55.

17. J. Slane, J. Vivanco, W. Rose, H.-L. Ploeg, M. Squire, Materials Science and Engineering: C 48, 188-196. (2015).

18. R. de Lima, A.B. Seabra, N. Duran, J Appl Toxicol 32, 867-79. (2012).

19. M.S. Jackson, J. Bagg, M.N. Gupta, R.D. Sturrock, Rheumatology (Oxford) 38, 572-5. (1999).

20. A.J. Singer, J Dent Res 31, 591-7. (1952). 